\title{
ParFDA for Instance Selection for Statistical Machine Translation
}

\author{
Ergun Biçici \\ ergunbicici@yahoo.com \\ bicici.github.com
}

\begin{abstract}
We build parallel feature decay algorithms (ParFDA) Moses statistical machine translation (SMT) systems for all language pairs in the translation task at the first conference on statistical machine translation (Bojar et al., 2016a) (WMT16). ParFDA obtains results close to the top constrained phrase-based SMT with an average of 2.52 BLEU points difference using significantly less computation for building SMT systems than the computation that would be spent using all available corpora. We obtain BLEU bounds based on target coverage and show that ParFDA results can be improved by 12.6 BLEU points on average. Similar bounds show that top constrained SMT results at WMT16 can be improved by 8 BLEU points on average while German to English and Romanian to English translations results are already close to the bounds.
\end{abstract}

\section{ParFDA}

ParFDA (Biçici et al., 2015) is a parallel implementation of feature decay algorithms (FDA), a class of instance selection algorithms that use feature decay, developed for fast deployment of accurate SMT systems. We use ParFDA for selecting parallel training data and language model (LM) data for building SMT systems. ParFDA runs separate FDA5 (Biçici and Yuret, 2015) models on randomized subsets of the available data and combines the selections afterwards. ParFDA allows rapid prototyping of SMT systems for a given target domain or task. FDA pseudocode is in Figure 1. This year, we have kept record of which 1gram or 2-grams of the test set have already been

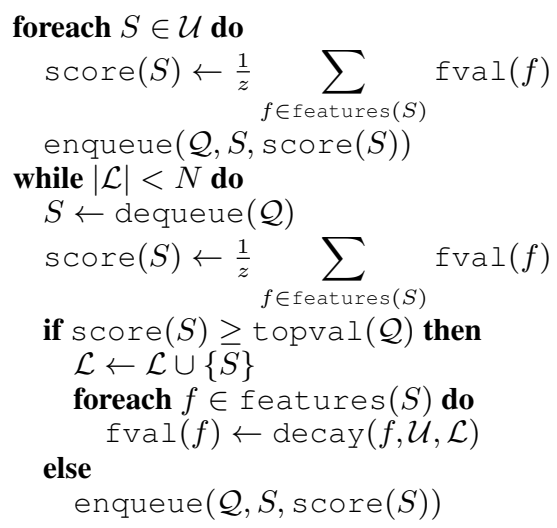

Figure 1: The Feature Decay Algorithm: inputs are a sentence pool $\mathcal{U}$, test set features $\mathcal{F}$, and number of instances to select $N$ and a priority queue $\mathcal{Q}$ stores sentence, $S$, scores score that sums feature values fval.

included to include an instance if otherwise found and we also use numeric expression identification using regular expressions to replace them with a label (Biçici, 2016) before instance selection.

We run ParFDA SMT experiments using Moses (Koehn et al., 2007) for all language pairs in both directions in the WMT16 translation task (Bojar et al., 2016a), which include English-Czech (en-cs), English-German (en-de), English-Finnish (en-fi), English-Romanian (enro), English-Russian (en-ru), and English-Turkish (en-tr).

\section{ParFDA Moses SMT Experiments}

The importance of ParFDA increases with the proliferation of training resources available for building SMT systems. Compared with WMT15 (Bojar et al., 2015), WMT16 observed significant increase in monolingual and parallel training data made available. Table 1 presents the statistics of the available training and LM corpora for the 


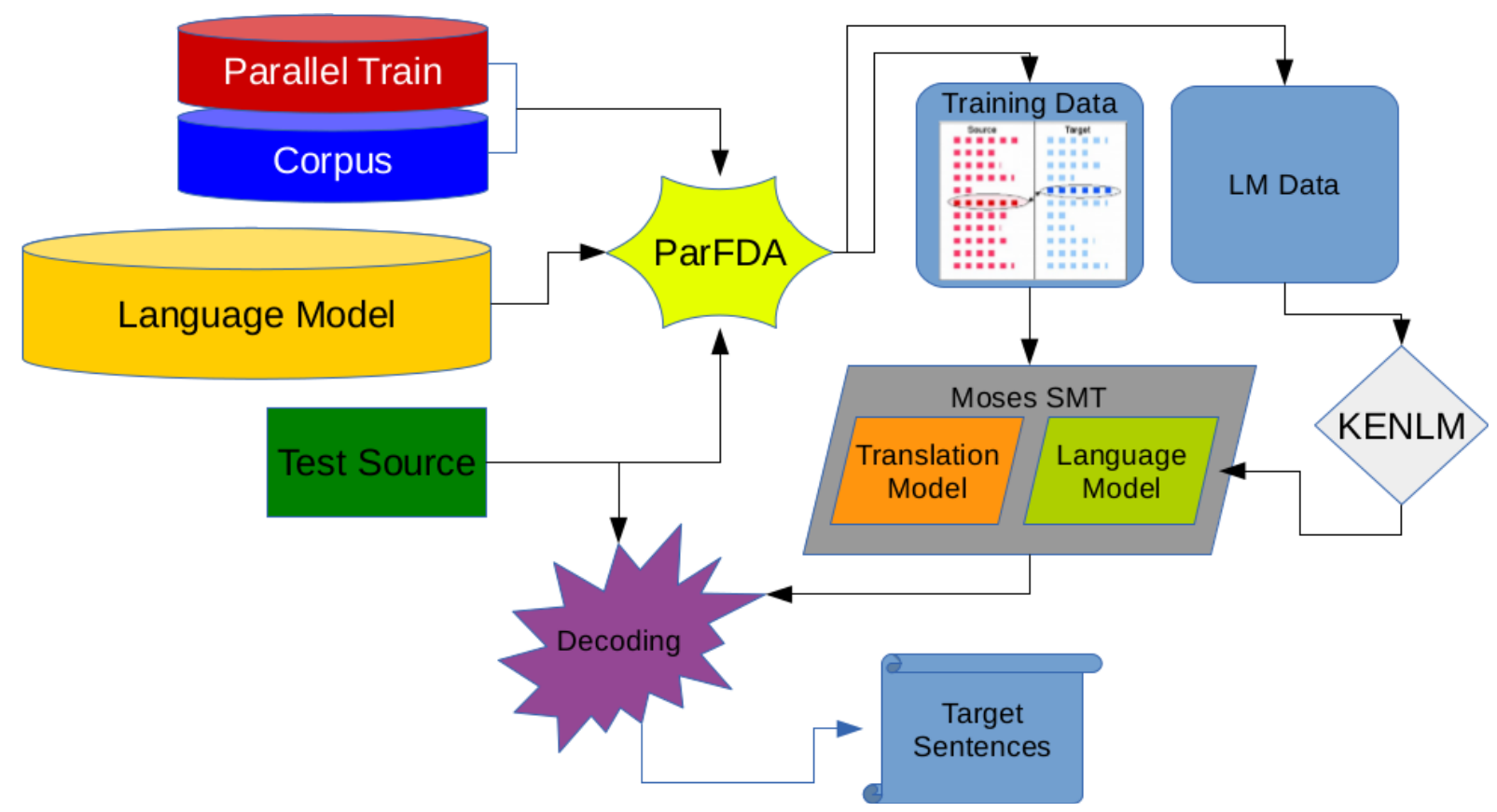

Figure 2: ParFDA Moses SMT workflow.

constrained (C) systems in WMT16 (Bojar et al., 2016a) as well as the statistics of the ParFDA selected subset training and LM data from C. TCOV lists the target coverage in terms of the 2-grams of the test set. Compared with last year, this year we do not use Common Crawl parallel corpus except for en-ru. We use Common Crawl monolingual corpus fi, ro, and tr datasets and we extended the LM corpora with previous years' corpora. We also use CzEng16pre (Bojar et al., 2016b) for en-cs.

We have increased the size of the training data selected to about 1.6 million instances to help with the reduction of out-of-vocabulary items. Except for translation directions involving Romanian and Turkish, this corresponds to increased training set size compared with ParFDA experiments in 2015, where we were able to obtain the top translation error rate (TER) performance in French to English translation using 1.261 million training sentences (Biçici et al., 2015). Due to the presence of peaks in SMT performance with increasing training set size (Biçici and Yuret, 2015), increasing the training set size need not improve the performance. We select about 15 million sentences for each LM not including the selected training set, which is added later. Table 1 shows the significant size differences between the constrained dataset (C) and the ParFDA selected data. We use 3-grams for selecting training data and 2-grams for LM corpus selection. Task specific data selection also im- proves the LM perplexity and the performance of the selected LM can be observed in Table 4 .

We truecase all of the corpora, set the maximum sentence length to 126 , use 150-best lists during tuning, set the LM order to 6 for all language pairs, and train the LM using KENLM (Heafield et al., 2013). For word alignment, we use mgiza (Gao and Vogel, 2008) where GIZA++ (Och and Ney, 2003) parameters set max-fertility to 10 , the number of iterations to 7,3,5,5,7 for IBM models $1,2,3,4$, and the HMM model, and learn 50 word classes in three iterations with the mkcls tool during training. The development set contains up to 3000 sentences randomly sampled from previous years' development sets (2011-2015) and remaining come from the development set for WMT16. ParFDA Moses SMT workflow is depicted in Figure 2.

ParFDA Moses SMT results for each translation direction at WMT16 are in Table 2 using BLEU over cased text, and $F_{1}$ (Biçici, 2011). We compare ParFDA results with the top constrained submissions at WMT16 in Table 3. ${ }^{1}$ The average difference to the top constrained (TopC) submission in WMT16 is 5.26 BLEU points whereas the difference was 3.2 BLEU points in WMT15 (Biçici et al., 2015). Performance compared with the TopC phrase-based SMT improved over WMT15 results with 2.52 BLEU points difference on av-

\footnotetext{
${ }^{1}$ We use the results from matrix. statmt. org.
} 


\begin{tabular}{lc|rrrl|rl}
\hline \multirow{S}{S}{$\rightarrow T$} & \multicolumn{4}{|c|}{ Training Data } & \multicolumn{2}{c}{ LM Data } \\
& Data & \#word S (M) & \#word T (M) & \#sent (K) & TCOV & \#word (M) & TCOV \\
\hline en-cs & C & 743.4 & 635.9 & 55025 & 0.741 & 1375.4 & 0.851 \\
en-cs & ParFDA & 70.5 & 59.2 & 1904 & 0.652 & 342.6 & 0.794 \\
cs-en & C & 743.4 & 635.9 & 55025 & 0.848 & 4859.0 & 0.945 \\
cs-en & ParFDA & 65.4 & 71.7 & 1906 & 0.788 & 436.2 & 0.904 \\
\hline en-de & C & 116.8 & 110.3 & 4513 & 0.706 & 2393.0 & 0.879 \\
en-de & ParFDA & 59.9 & 54.2 & 1701 & 0.683 & 368.7 & 0.824 \\
de-en & $\mathrm{C}$ & 116.8 & 110.3 & 4513 & 0.793 & 4859.0 & 0.945 \\
de-en & ParFDA & 55.7 & 56.3 & 1692 & 0.772 & 422.6 & 0.901 \\
\hline en-fi & $\mathrm{C}$ & 52.6 & 37.7 & 2026 & 0.406 & 2971.8 & 0.744 \\
en-fi & ParFDA & 49.1 & 35.0 & 1637 & 0.404 & 492.1 & 0.657 \\
fi-en & $\mathrm{C}$ & 52.6 & 37.7 & 2026 & 0.666 & 4859.0 & 0.935 \\
fi-en & ParFDA & 35.0 & 48.6 & 1626 & 0.662 & 413.5 & 0.888 \\
\hline en-ro & $\mathrm{C}$ & 15.7 & 16.0 & 597 & 0.596 & 8067.4 & 0.932 \\
en-ro & ParFDA & 15.7 & 16.0 & 597 & 0.596 & 615.6 & 0.871 \\
ro-en & $\mathrm{C}$ & 15.7 & 16.0 & 597 & 0.681 & 4859.0 & 0.948 \\
ro-en & ParFDA & 16.0 & 15.7 & 597 & 0.681 & 381.6 & 0.909 \\
\hline en-ru & $\mathrm{C}$ & 51.7 & 48.4 & 2570 & 0.668 & 1038.7 & 0.866 \\
en-ru & ParFDA & 44.3 & 40.1 & 1654 & 0.664 & 355.3 & 0.826 \\
ru-en & $\mathrm{C}$ & 51.7 & 48.4 & 2570 & 0.794 & 4859.0 & 0.951 \\
ru-en & ParFDA & 40.6 & 43.4 & 1643 & 0.788 & 410.8 & 0.912 \\
\hline en-tr & $\mathrm{C}$ & 5.1 & 4.6 & 206 & 0.352 & 11674.3 & 0.848 \\
en-tr & ParFDA & 5.1 & 4.6 & 205 & 0.352 & 545.0 & 0.729 \\
tr-en & $\mathrm{C}$ & 5.1 & 4.6 & 206 & 0.56 & 4859.0 & 0.934 \\
tr-en & ParFDA & 4.6 & 5.1 & 205 & 0.56 & 371.2 & 0.887 \\
\hline & & & & & & &
\end{tabular}

Table 1: Data statistics for the available training and LM corpora in the constrained (C) setting compared with the ParFDA selected training and LM data. \#words is in millions (M) and \#sents in thousands (K). TCOV is target 2 -gram coverage.

\begin{tabular}{l|llllll|llllll} 
& \multicolumn{6}{|c|}{$S \rightarrow$ en } & \multicolumn{7}{c}{$e n \rightarrow T$} \\
& cs-en & de-en & fi-en & ro-en & ru-en & tr-en & en-cs & en-de & en-fi & en-ro & en-ru & en-tr \\
\hline BLEU & 0.2641 & 0.3014 & 0.1744 & 0.2904 & 0.2525 & 0.1222 & 0.1942 & 0.2391 & 0.1248 & 0.2097 & 0.2193 & 0.0901 \\
$F_{1}$ & 0.2718 & 0.3067 & 0.2077 & 0.289 & 0.2674 & 0.1641 & 0.2169 & 0.2592 & 0.1665 & 0.2258 & 0.2363 & 0.1346 \\
\hline
\end{tabular}

Table 2: ParFDA results at WMT16.

erage, which is likely due to selecting increased number of training data.

We observe that various systems in TopC used character-level split and merge operations (referred as BPE or byte pair encoding) combined with neural networks (Sennrich et al., 2016). ${ }^{2}$ We also compare ParFDA results with the TopC $\mathrm{BPE}$ and the average difference is 5.86 BLEU points. ${ }^{3}$ WMT15 did not contain any submission with BPE. Average difference between TopC $\mathrm{BPE}$ and TopC phrase hints that majority of the in-

\footnotetext{
${ }^{2}$ For instance within en-de translation results: matrix. statmt.org/matrix/systems_list/1840.

${ }^{3}$ Some translation directions did not contain BPE results.
}

creased performance difference is due to improvements obtained by BPE in TopC BPE results.

Table 4 compares the perplexity of the ParFDA selected LM with a LM trained on the ParFDA selected training data and a LM trained using all of the available training corpora and shows reductions in the number of OOV tokens reaching up to $45 \%$ and the perplexity up to $45 \%$. Table 4 also presents the average log probability of tokens and the $\log$ probability of token $<$ unk $>$ returned by KENLM to token $<u n k>$. The increase in the ratio between them in the last column shows that OOV in ParFDA LM are not just less but also less likely at the same time. 


\begin{tabular}{|c|c|c|c|c|c|c|c|c|c|c|c|c|}
\hline BLEU & cs-en & de-en & fi-en & ro-en & ru-en & tr-en & en-cs & en-de & en-fi & en-ro & en-ru & en-tr \\
\hline $\begin{array}{l}\text { ParFDA } \\
\end{array}$ & 0.2641 & 0.3014 & 0.1744 & 0.2904 & 0.2525 & 0.1222 & 0.1942 & 0.2391 & 0.1248 & 0.2097 & 0.2193 & 0.0901 \\
\hline TорС & 0.314 & 0.386 & 0.204 & 0.352 & 0.291 & 0.145 & 0.258 & 0.342 & 0.174 & 0.289 & 0.26 & 0.098 \\
\hline - ParFDA & 0.0499 & 0.0846 & 0.0296 & 0.0616 & 0.0385 & 0.0228 & 0.0638 & 0.1029 & 0.0492 & 0.0793 & 0.0407 & 0.0079 \\
\hline avg diff & 0.0526 & & & & & & & & & & & \\
\hline TopC BPE & 0.314 & 0.386 & & 0.339 & 0.291 & & 0.258 & 0.342 & 0.151 & 0.282 & 0.26 & \\
\hline - ParFDA & 0.0499 & 0.0846 & & 0.0486 & 0.0385 & & 0.0638 & 0.1029 & 0.0262 & 0.0723 & 0.0407 & \\
\hline avg diff & 0.0586 & & & & & & & & & & & \\
\hline TopC phrase & 0.304 & 0.345 & 0.191 & 0.322 & 0.27 & 0.129 & 0.236 & 0.283 & 0.138 & 0.235 & 0.24 & 0.092 \\
\hline - ParFDA & 0.0399 & 0.0436 & 0.0166 & 0.0316 & 0.0175 & 0.0068 & 0.0418 & 0.0439 & 0.0132 & 0.0253 & 0.0207 & 0.0019 \\
\hline avg diff & 0.0252 & & & & & & & & & & & \\
\hline $\begin{array}{l}\text { BPE - phrase } \\
\text { avg diff }\end{array}$ & $\begin{array}{l}0.01 \\
0.0278\end{array}$ & 0.041 & & 0.017 & 0.021 & & 0.022 & 0.059 & 0.013 & 0.047 & 0.02 & \\
\hline
\end{tabular}

Table 3: ParFDA results compared with the top constrained results in WMT16 (TopC, from matrix . statmt.org) and their difference.

\begin{tabular}{|c|c|c|c|c|c|c|c|c|c|c|c|c|c|c|c|}
\hline \multirow[b]{2}{*}{$S \rightarrow T$} & \multicolumn{4}{|c|}{ OOV Rate } & \multicolumn{4}{|c|}{ perplexity } & \multicolumn{3}{|c|}{ avg log prob. } & \multicolumn{3}{|c|}{$<$ unk $>$ log prob. } & \multirow{2}{*}{$\begin{array}{l}\frac{\langle\text { unk }>}{\text { avg }} \\
\text { \%inc }\end{array}$} \\
\hline & $\begin{array}{c}\mathrm{C} \\
\text { train }\end{array}$ & $\begin{array}{c}\text { FDA5 } \\
\text { train }\end{array}$ & $\begin{array}{c}\text { FDA5 } \\
\text { LM }\end{array}$ & \%red & $\underset{\text { train }}{\mathrm{C}}$ & $\begin{array}{c}\text { FDA5 } \\
\text { train }\end{array}$ & $\begin{array}{c}\text { FDA5 } \\
\text { LM }\end{array}$ & \%red & $\begin{array}{c}\mathrm{C} \\
\text { train }\end{array}$ & $\begin{array}{c}\text { FDA5 } \\
\text { train }\end{array}$ & $\begin{array}{c}\text { FDA5 } \\
\text { LM }\end{array}$ & $\underset{\text { train }}{\mathrm{C}}$ & $\begin{array}{c}\text { FDA5 } \\
\text { train }\end{array}$ & $\begin{array}{c}\text { FDA5 } \\
\text { LM }\end{array}$ & \\
\hline en-cs & 0.259 & 0.299 & 0.256 & 0.01 & 14946 & 11609 & 9428 & 0.37 & -4.61 & -4.57 & -4.39 & -7.8 & -7.11 & -7.77 & 0.05 \\
\hline en-de & 0.361 & 0.372 & 0.28 & 0.22 & 7075 & 6217 & 4297 & 0.39 & -4.28 & -4.23 & -3.94 & -7.31 & -7.08 & -7.77 & 0.16 \\
\hline en-fi & 0.409 & 0.412 & 0.237 & 0.42 & 45087 & 49807 & 27698 & 0.39 & -5.67 & -5.73 & -4.96 & -7.04 & -7.0 & -8.15 & 0.32 \\
\hline en-ro & 0.389 & 0.389 & 0.239 & 0.39 & 3043 & 3043 & 2150 & 0.29 & -3.92 & -3.92 & -3.58 & -6.35 & -6.35 & -7.87 & 0.36 \\
\hline en-ru & 0.317 & 0.319 & 0.288 & 0.09 & 10245 & 10787 & 8555 & 0.16 & -4.55 & -4.58 & -4.41 & -7.16 & -7.09 & -7.74 & 0.12 \\
\hline en-tr & 0.416 & 0.416 & 0.229 & 0.45 & 18988 & 18988 & 15805 & 0.17 & -5.14 & -5.14 & -4.63 & -6.18 & -6.18 & -8.09 & 0.45 \\
\hline cs-en & 0.285 & 0.336 & 0.27 & 0.05 & 2647 & 2095 & 1549 & 0.41 & -3.64 & -3.58 & -3.38 & -7.54 & -6.88 & -7.58 & 0.08 \\
\hline de-en & 0.352 & 0.37 & 0.279 & 0.21 & 2521 & 2263 & 1426 & 0.43 & -3.69 & -3.65 & -3.36 & -7.1 & -6.87 & -7.58 & 0.17 \\
\hline fi-en & 0.41 & 0.419 & 0.274 & 0.33 & 2753 & 2972 & 1509 & 0.45 & -3.77 & -3.81 & -3.38 & -6.57 & -6.49 & -7.55 & 0.28 \\
\hline ro-en & 0.418 & 0.418 & 0.282 & 0.33 & 2017 & 2017 & 1422 & 0.29 & -3.66 & -3.66 & -3.37 & -6.24 & -6.24 & -7.54 & 0.31 \\
\hline ru-en & 0.352 & 0.358 & 0.291 & 0.17 & 1907 & 1974 & 1532 & $0.2^{2}$ & -3.55 & -3.57 & -3.4 & -6.98 & -6.89 & -7.58 & 0.13 \\
\hline tr-en & 0.466 & 0.466 & 0.297 & 0.36 & 2250 & 2250 & 1584 & 0.3 & -3.73 & -3.73 & -3.42 & -5.98 & -5.98 & -7.54 & 0.38 \\
\hline
\end{tabular}

Table 4: Perplexity comparison of the LM built from the training corpus (train), ParFDA selected training data (FDA5 train), and the ParFDA selected LM data (FDA5 LM). \%red is proportion of reduction and prob. is used for probability.

\section{Translation Upper Bounds with TCOV}

In this section, we obtain upper bounds on the translation performance based on the target coverage (TCOV) of $n$-grams of the test set found in the selected ParFDA training data. We obtain translations based on TCOV by randomly replacing some number of tokens from a given sentence with a fixed OOV label proportional to TCOV starting from 1-grams. After OOVs for 1-grams are identified, OOV tokens for $n$-grams up to 5 -grams are identified and BLEU is calculated with respect to the original. If the overall number of OOVs obtained before $i$-grams are enough to obtain the $i$ gram TCOV, then OOV identification for $i$-grams is skipped. Number of OOV tokens is identified by two possible functions for a given sentence $T^{\prime}$ :

$$
\begin{aligned}
& O O V_{r}=\operatorname{round}\left((1-\mathrm{TCOV}) *\left|T^{\prime}\right|\right) \\
& O O V_{f}=\left\lfloor(1-\mathrm{TCOV}) *\left|T^{\prime}\right|\right\rfloor
\end{aligned}
$$

where $\left|T^{\prime}\right|$ denotes the length of the sentence in the number of tokens.
We obtain each bound using 10000 such instances and repeat for 10 times. This TCOV BLEU bound is optimistic since it does not consider reorderings in the translation or differences in sentence length. Each plot in Tables 6 and 7 locates TCOV BLEU bound obtained from each $n$-gram and from $n$-grams combined up to and including $n$ and locates the ParFDA Moses SMT performance.

Table 5 compares TCOV BLEU bounds with ParFDA results and TopC from Table 3 and shows potential improvements in the translation performance for all translation directions at WMT16 and overall on average. Results in bold are close to $O O V_{r}$ TCOV BLEU bound, which indicates that TopC translation results for de-en and ro-en directions are able to obtain results close to this bound.

\section{Conclusion}

We use ParFDA for selecting instances for building SMT systems using less computation over- 


\begin{tabular}{|c|c|c|c|c|c|c|c|c|c|c|c|c|}
\hline BLEU & cs-en & de-en & fi-en & ro-en & ru-en & tr-en & en-cs & en-de & en-fi & en-ro & en-ru & en-tr \\
\hline ParFDA bound & 0.4501 & 0.3846 & 0.3516 & 0.3391 & 0.3968 & 0.3053 & 0.3292 & 0.3575 & 0.2415 & 0.3275 & 0.3383 & 0.1723 \\
\hline$\therefore \quad$ - ParFDA & 0.186 & 0.0832 & 0.1772 & 0.0487 & 0.1443 & 0.1831 & 0.135 & 0.1184 & 0.1167 & 0.1178 & 0.119 & 0.0822 \\
\hline avg diff & 0.126 & & & & & & & & & & & \\
\hline C BLEU bound & 0.4908 & 0.3864 & 0.3518 & 0.3392 & 0.3969 & 0.3054 & 0.3679 & 0.3572 & 0.2416 & 0.3274 & 0.3381 & 0.1719 \\
\hline $\begin{array}{c}- \text { TopC } \\
\text { avg diff }\end{array}$ & $\begin{array}{l}0.1768 \\
0.0801\end{array}$ & 0.0004 & 0.1478 & -0.0128 & 0.1059 & 0.1604 & 0.1099 & 0.0152 & 0.0676 & 0.0384 & 0.0781 & 0.0739 \\
\hline ParFDA bound & 0.4766 & 0.4143 & 0.3729 & 0.3842 & 0.4337 & 0.3072 & 0.3792 & 0.3704 & 0.2382 & 0.3416 & 0.3768 & 0.2283 \\
\hline $\begin{array}{l}- \text { ParFDA } \\
\text { avg diff }\end{array}$ & $\begin{array}{l}0.2125 \\
0.1534\end{array}$ & 0.1129 & 0.1985 & 0.0938 & 0.1812 & 0.185 & 0.185 & 0.1313 & 0.1134 & 0.1319 & 0.1575 & 0.1382 \\
\hline C BLEU bound & 0.5344 & 0.4156 & 0.3719 & 0.3718 & 0.4337 & 0.3068 & 0.3945 & 0.3847 & 0.2384 & 0.3411 & 0.3769 & 0.2005 \\
\hline $\begin{array}{c}- \text { TopC } \\
\text { avg diff }\end{array}$ & $\begin{array}{l}0.2204 \\
0.1048\end{array}$ & 0.0296 & 0.1679 & 0.0198 & 0.1427 & 0.1618 & 0.1365 & 0.0427 & 0.0644 & 0.0521 & 0.1169 & 0.1025 \\
\hline
\end{tabular}

Table 5: 1,2,3,4,5-gram TCOV BLEU bounds compared with WMT16 results. bold are close to a bound.

all than the computation that would be spent using all available corpora while still achieve SMT performance that is close to the top performing phrase-based SMT systems. ParFDA results at WMT16 provides new results using the current phrase-based SMT technology towards rapid SMT system development in budgeted training scenarios. ParFDA works towards the development of task or data adaptive SMT solutions using specially moulded data rather than general purpose SMT systems built with a patchwork approach combining various sources of information and several processing steps.

We obtain BLEU bounds based on target coverage and show that top constrained results can be improved by 8 BLEU points on average and obtain results close to the bound for de-en and ro-en translation directions. Similar bounds show that ParFDA results can be improved by 12.6 BLEU points on average.

\section{Acknowledgments}

We thank the reviewers for providing constructive comments.

\section{References}

Ergun Biçici and Deniz Yuret. 2015. Optimizing instance selection for statistical machine translation with feature decay algorithms. IEEE/ACM Transactions On Audio, Speech, and Language Processing (TASLP), 23:339-350.

Ergun Biçici, Qun Liu, and Andy Way. 2015. ParFDA for fast deployment of accurate statistical machine translation systems, benchmarks, and statistics. In Proc. of the EMNLP 2015 Tenth Workshop on Statistical Machine Translation, Lisbon, Portugal, 9. Association for Computational Linguistics.

Ergun Biçici. 2011. The Regression Model of Machine
Translation. Ph.D. thesis, Koç University. Supervisor: Deniz Yuret.

Ergun Biçici. 2016. RTM at SemEval-2016 task 1: Predicting semantic similarity with referential translation machines and related statistics. In SemEval-2016: Semantic Evaluation Exercises - International Workshop on Semantic Evaluation, San Diego, USA, 6.

Ondrej Bojar, Rajan Chatterjee, Christian Federmann, Barry Haddow, Chris Hokamp, Matthias Huck, Pavel Pecina, Philipp Koehn, Christof Monz, Matteo Negri, Matt Post, Carolina Scarton, Lucia Specia, and Marco Turchi. 2015. Findings of the 2015 workshop on statistical machine translation. In Proc. of the Tenth Workshop on Statistical Machine Translation, Lisbon, Portugal, September.

Ondrej Bojar, Christian Buck, Rajan Chatterjee, Christian Federmann, Liane Guillou, Barry Haddow, Matthias Huck, Antonio Jimeno Yepes, Aurlie Nvol, Mariana Neves, Pavel Pacina, Martin Poppel, Philipp Koehn, Christof Monz, Matteo Negri, Matt Post, Lucia Specia, Karin Verspoor, Jrg Tiedemann, and Marco Turchi. 2016a. Proc. of the 2016 conference on statistical machine translation. In Proc. of the First Conference on Statistical Machine Translation (WMT16), Berlin, Germany, August.

Ondřej Bojar, Ondřej Dušek, Tom Kocmi, Jindřich Libovický, Michal Novák, Martin Popel, Roman Sudarikov, and Dušan Variš. 2016b. CzEng 1.6: Enlarged Czech-English Parallel Corpus with Processing Tools Dockered. In Text, Speech and Dialogue: 19th International Conference, TSD 2016, Brno, Czech Republic, September 12-16, 2016, Proceedings. Springer Verlag, September 12-16. In press.

Qin Gao and Stephan Vogel, 2008. Software Engineering, Testing, and Quality Assurance for Natural Language Processing, chapter Parallel Implementations of Word Alignment Tool, pages 49-57. Association for Computational Linguistics.

Kenneth Heafield, Ivan Pouzyrevsky, Jonathan H. Clark, and Philipp Koehn. 2013. Scalable modified Kneser-Ney language model estimation. In Proc. of 

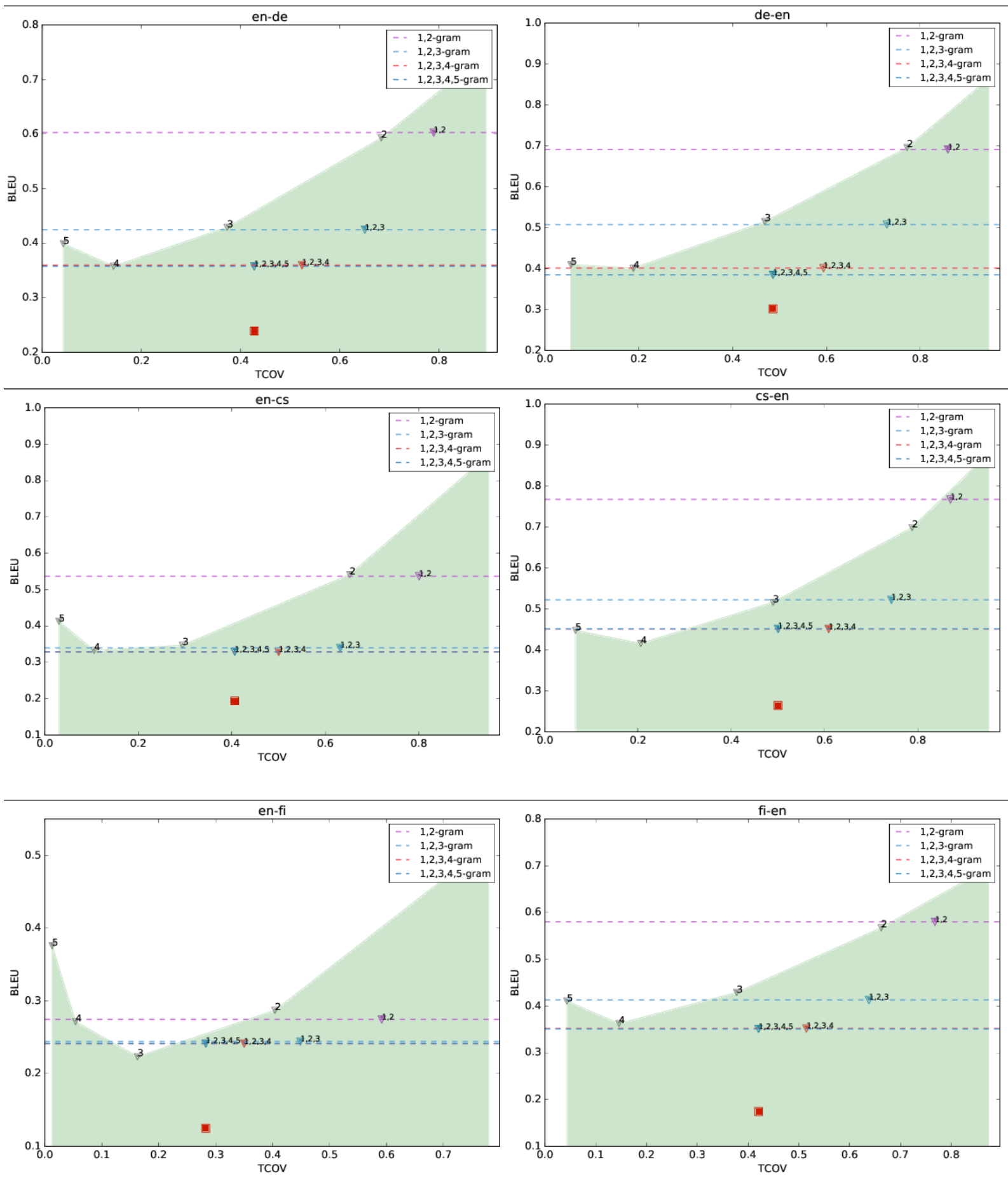

Table 6: ParFDA results ( $\square)$ and $O O V_{r}$ TCOV BLEU upper bounds for cs, de, and fi.

the 51st Annual Meeting of the Association for Computational Linguistics, pages 690-696, Sofia, Bulgaria, August.

Philipp Koehn, Hieu Hoang, Alexandra Birch, Chris Callison-Burch, Marcello Federico, Nicola Bertoldi, Brooke Cowan, Wade Shen, Christine Moran, Richard Zens, Chris Dyer, Ondrej Bojar, Alexandra Constantin, and Evan Herbst. 2007. Moses: Open source toolkit for statistical machine translation. In Proc. of the 45th Annual Meeting of the Association for Computational Linguistics Companion Volume Proc. of the Demo and Poster Sessions, pages 177180. Association for Computational Linguistics.

Franz Josef Och and Hermann Ney. 2003. A systematic comparison of various statistical alignment models. Computational Linguistics, 29(1):19-51.

Rico Sennrich, Barry Haddow, and Alexandra Birch. 2016. Edinburgh neural machine translation systems for wmt 16. In Proc. of the ACL 2016 

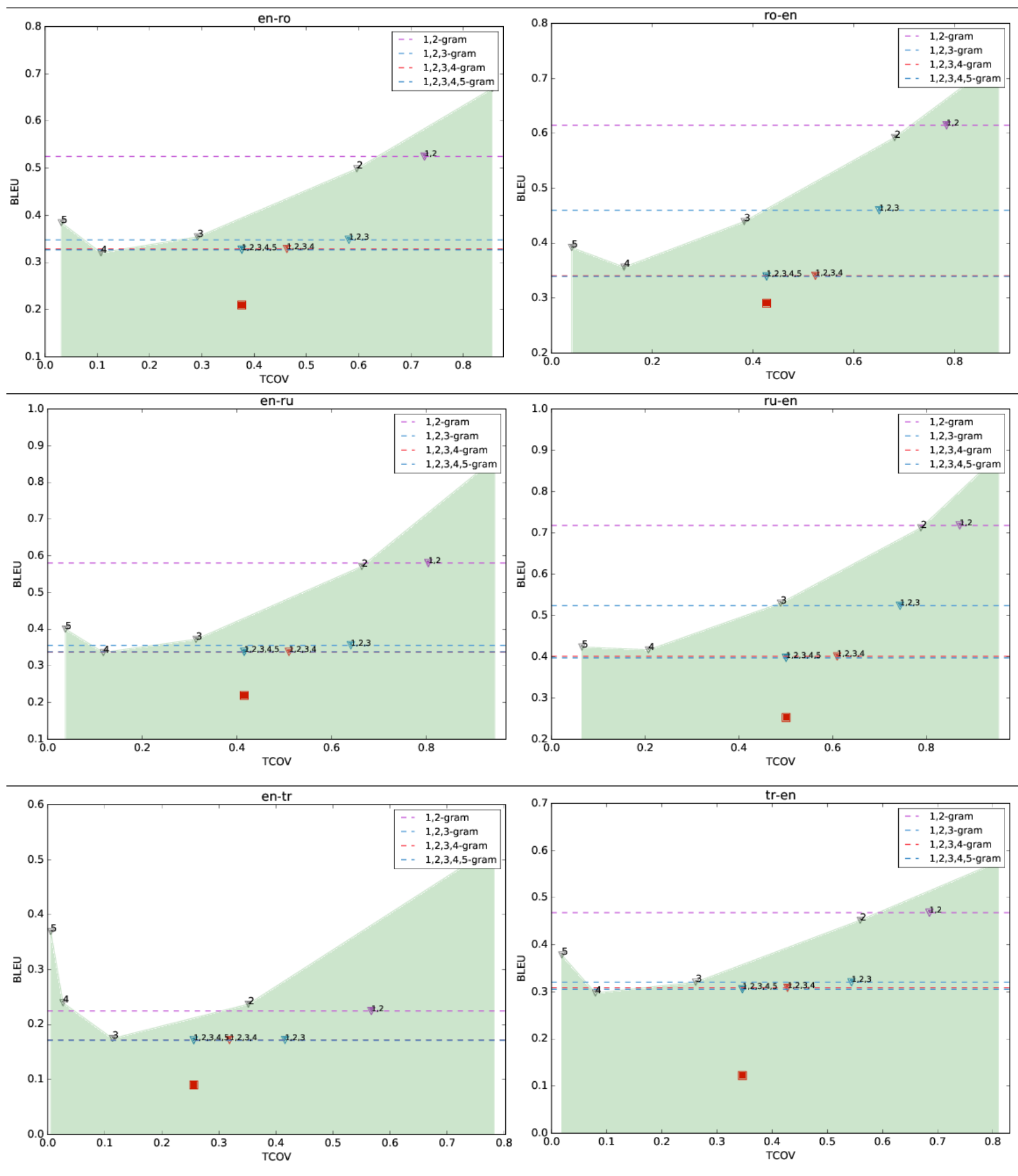

Table 7: ParFDA results ( $\square$ ) and $O O V_{r}$ TCOV BLEU upper bounds for ro, ru, and tr.

Eleventh Workshop on Statistical Machine Translation, Berlin, Germany, 8. Association for Computational Linguistics. 